Instead they strongly support a simple two-state stacked-unstacked equilibrium.

As to optical methods, and the assessment of stacking from such measurements, Cantor and Tinoco (Biopolymers, 5, 821; 1967) have compared calculated and observed optical rotatory dispersion curves for nine trimers, seven of them trinucleoside diphosphates (no terminal phosphate). It is confirmed that there is good agreement on the basis of sums of pairwise interactions along the chain, and this work supports the validity of analyses of single-stranded polynucleotides in these simple terms. At the same time, Inoue et al. (J. Amer. Chem. Soc., 89, 5701; 1967) have looked at seven trinucleotides-all with the terminal phosphate group-and obtain less satisfactory agreement with calculated curves; it can be deduced that there is a considerable effect from the terminal charge. It is also found that stacking persists in several cases at alkaline $p H$, so long as not more than one of the bases in the trimer becomes ionized.

\section{Making Safe Vaccines}

\section{from a Correspondent}

AT the fourth meeting of the Cell Culture Committee of the Permanent Section of Microbiological Standardization, held at the National Institute for Medical Research (Hampstead Laboratories) in September last year, much attention was given to the measures that can be taken to eliminate hazards encountered in virus vaccine production, in particular the elimination of oncogenic factors in the final vaccine. It was emphasized that more standardization both of media and of cell substrates would do much to achieve this goal.

As far as cell substrates are concerned, the tissue used on the widest scale is grown from monkey kidney. The primary tissue is not tested for the presence of extraneous agents although attempts are made to isolate viruses from control cultures throughout the period of vaccine production. The recent episode in which a number of laboratory personnel werc infected by contact with the blood or tissue of a group of African green monkeys tragically underlines the dangers of using primary cultures of uncharacterized tissues. The further possibility cannot be overlooked that an undetected infectious agent may survive in the final vaccine product and become apparent only after injection into man.

Other primary tissues used for vaccine production are derived from chick and duck embryos and canine kidney. These animals are obviously bred in a much cleaner environment than obtains for monkeys in the wild, but the possible presence of extraneous agents nevertheless enjoins caution. Thus although chickens have been bred under controlled conditions which are free from fowl leukosis viruses, a new infectious agentthat of Marek's disease-has recently been isolated and only a few of the commercial flocks are free from both agents. Rabbit kidney and guinea-pig kidney are also now being used on a small scale; the absence of reported isolations of extraneous agents from primary cultures of these tissues may be due to lack of experience with these cultures rather than freedom from infection.

Any new cell substrate for virus vaccine production must be derived from normal tissue and must remain normal throughout its propagation. The cells must be frozen until they have been cleared for use by the necessary exhaustive tests. It is also necessary that such tissues should be suitable for the growth of human viruses in high titre.

Objections have been raised to the use of human tissue on the grounds that an undetected agent (oncogenic virus or "slow" virus) will be more dangerous in a vaccine to be used in man than similar viruses that may occur in animal tissue. It must, however, be pointed out that none of the primate oncogenic viruses $\left(S V_{40}\right.$ and adenoviruses) gives rise to tumours in the species in which it is indigenous, although such viruses do produce tumours in other animal species (hamsters and the like). Furthermore, "slow" viruses are known to cross species barriers (scrapie in sheep and mice), and animal tissue would therefore not be safer than human tissue in this respect.

The committee was particularly anxious that greater attention should be paid to the use of standardized cell culture media. The use of a powdered medium made in a large batch and tested for suitability before release has done much to minimize changes that can arise as a result of nutritionally deficient or drastically altered medium. Most antibiotics, although preventing the growth of bacteria, have no effect on the growth of some strains of mycoplasma and their use provides no assurance against mycoplasma contamination. Some batches of serum presumed sterile have been found to be contaminated; they may vary widely in growth promoting activity, and changing batches may occasionally cause chromosomal changes in a cell with an unstable chromosomal complement.

For obvious reasons, much attention is being given to the potential oncogenicity in man of new cell substrates. It has been suggested, for example, that a latent viral genome in the tissue may hybridize with the virus used for vaccine production and thereby be activated. In general, animal inoculation tests are insensitive for the detection of oncogenic viruses, and in vitro tests are urgently needed. The fusion of candidate cells for virus production with a variety of other cell types may provide a useful tool for the demonstration, or exclusion, of the presence of incomplete virus in such tissues.

The hazards of making virus vaccines stem primarily from the use of ill defined and potentially contaminated substrates. It is encouraging to learn of the increasing activity in the use of a well characterized human cell (WI-38) in the preparation of vaccines for clinical trial and to learn also of their use, without incident, in a total of well over a million human subjects.

\section{Muscle Meeting}

\section{from a Correspondent}

PAssers-By in Gower Street just before Christmas, seeing the large notices proclaiming the "Muscle Meeting", might have been forgiven for supposing that this was a congress of weightlifters. It was in fact merely the winter meeting of the British Biophysical Society: this bore the title "Muscular Con. traction" and was organized by Dr H. E. Huxley and Professor D. R. Wilkie. There was an unusually full 